seems improbable that we could have overlooked more than two such events.

It is of interest that the smallest velocity of the a-particle which has thus far been sufficient to disintergrate a nitrogen atom in the work at Cambridge and in Chicago corresponds to a remaining range at I $5^{\circ}$ of about $6.2 \mathrm{~cm}$. Now the range of an $a$-particle from polonium, which was used in practically all of the early work on a-ray tracks, is only $3.925 \mathrm{~cm}$. It is evident that if polonium had been used as a source, not one of the ten disintegrations thus far found would have been obtained. It was because the early work on the scintillation method indicated such a result that Harkins and Ryan changed to a source which contained thorium $\mathrm{C}^{\prime}$, such as thorium $\mathrm{C}$, or thorium $\mathrm{B}$ and $\mathrm{C}$.

According to Petterson and Schmidt ("Atomzertrümmerung," p. Iog) the a-rays from polonium liberate $\mathrm{H}$-particles from aluminium, but the results cited above do not indicate this to be true in the case of nitrogen. However, it will be necessary to obtain more photographs, and to examine again those already obtained before a definite conclusion can be drawn.

A part of the measurements on the photograph (Fig. I) were obtained by a new method, which corrects for errors in the lenses and mirrors. It is equivalent to the establishment in the ionisation chamber of a set of co-ordinates in space.

We wish to thank Mr. C. N. Shah for a considerable part of the calculations involved, and for much time spent in the examination of the films.

WILLIAM D. HARKINS.

Hugh A. Shadduck.

\section{Quantum Mechanics and the Magnetic Moment of Atoms.}

IN a recent paper E. Schrödinger (Ann. d. Phys., 81, 1926, I09) put forward a hypothesis connecting the field scalar $\psi$ of his undulatory mechanics with the electric density of current. His formulæ in the case of a system with only one electron (expressing $j$ in electromagnetic units) reduce to :

$$
\left.\begin{array}{l}
\rho=e \psi \bar{\psi}, \\
j=\frac{h e}{4 \pi i m c}(\psi \operatorname{grad} . \bar{\psi}-\bar{\psi} \operatorname{grad} . \psi
\end{array}\right),
$$

where $\rho$ and $j$ are respectively the electric density and density of current. The field scalar must be normalised in such a way that

$$
\int \psi \bar{\psi} d \tau=\mathrm{I},
$$

$d \tau$ being the element of volume.

Evidence in favour of the expression for $\rho$ is given by the fact that the calculation of the intensity of emission of the atom, based on it, is in accordance both with experiment and with the results of Heisenberg's quantum mechanics. Expression (2) for $j$ is assumed by Schrödinger as the simplest way of satisfying the equation of continuity of electricity.

It is desirable, therefore, to find some further evidence for this expression. I propose to show that it leads to the right expression for the magnetic moment of a hydrogen-like atom. We will consider only the part of the magnetic moment which, in the old quantum theory, was supposed to be due to the orbital motion of the electron, not taking into account the magnetic moment of the spinning electron, which can be considered separately.

The wave equation for a hydrogen-like atom in a magnetic field (without considering the spinning

$$
\text { No. 298I, VOL. I I } 8]
$$

electron) was integrated by Fock (Zeit. $f$, Phys., 28, $1926,242)$. His expression for $\psi$ can be written in the form

$$
\psi=f(\theta, v) e^{i n_{1} \phi e^{2 \pi i} h^{t},},
$$

where $r, \theta, \phi$ are polar co-ordinates; $f(\theta, r)$ is a real function of $\gamma$ and $\theta ; n_{1}$ is an integer corresponding to the magnetic quantum number of the old theory.

It is easily seen from (2) and (4) that $j$ is a vector perpendicular at each point to the plane passing through the point and the polar axis, the magnitude of which is

$$
j=\frac{h e}{4 \pi i m c} \frac{I}{R}\left(\psi \frac{\partial \vec{\psi}}{\partial \phi}-\bar{\psi} \frac{\partial \psi}{\partial \phi}\right)=-\frac{h e}{2 \pi m c R} n_{1} f^{2}, .
$$

$R$ being the distance of the point from the axis. The lines of current are circles situated in planes perpendicular to the axis and with their centres on the axis.

Let us now consider the current passing through an element $d \sigma$ of a meridian plane; its intensity is given by $j d \sigma$ and its circuit is a circle with radius $R$. The magnetic moment of this current is therefore $\pi R^{2} j d \sigma$ (in the direction of the field).

The magnetic moment of the atom becomes, putting for $j$ the expression (5),

$$
\mu=\pi \int R^{2} j d \sigma=-\frac{h e n_{1}}{2 m c} \int R f^{2} d \sigma .
$$

The last integral can be valuated by substituting (4) in (3), and observing that $d \tau=2 \pi R d \sigma$; we find then $2 \pi \int R f^{2} d \sigma=\mathrm{I}$. Putting this value into (6) we find at last

$$
\mu=-\frac{h e}{4 \pi m c} n_{1} \text {. }
$$

That is, the component of the magnetic moment in the direction of the field is the product of a Bohr magneton $\left(-\frac{h e}{4 \pi m c}\right)$ and the magnetic quantum number $n_{1}$, as it was expected.

It is noteworthy that the magnetic moment (7) arises in a certain way through the action of the field. In the absence of a magnetic field, the two states with $n_{1}$ equal in absolute value but of opposite signs, have the same energy; every linear combination of these two states is therefore a quantum state. Now it is easily seen from (4) that we can combine the two states in such a way that $\psi$ becomes the product of $e^{2 \pi i} \frac{E}{h}$ and a real function of $r, \theta, \phi$ (containing $\cos n_{1} \phi$ or $\sin n_{1} \phi$, as a factor). In this case the density of current $j$ would vanish everywhere, and the magnetic moment would reduce to zero. ENRICO FERMi.

Physical Institute of the University, Rome, November I4.

\section{Psychical Phenomena and their Interpretation.}

Pursque une controverse courtoise a pris place dans ce journal au sujet des recherches psychiques, il me sera permis de vous adresser quelques mots au sujet de mon opinion, motivée par de longues recherches (voir mon "Traité de Métapsychique," traduit en anglais, 1925, "Thirty Years of Psychical Research ").

Il importe avant tout de distinguer les faits et les théories.

Pour les faits, ils ont été, à ce qu'il me semble, établis en toute évidence, et on ne peut attribuer ni à la fraude, ni à, l'erreur, ni à l'illusion, la constatation de la plupart des phénomènes. Encore, à l'extrème rigueur. peut-on soutenir que les faits d'ectoplasmie, de lumières. 